\title{
Evaluation of Electroencephalogram Signals of the Professional Pianists during Iconic Memory and Working Memory Tests Using Spectral Coherence
}

\begin{abstract}
Background: Today, the neuroscience has growth in many aspects, and the effects of different factors on memory obtained many achievements. Several scientific and experimental studies evaluated effects of music on style and behavior of people; in this study, we evaluated memory between two groups of people, the professional pianists and normal people, through processing their electroencephalogram (EEG) signals using the coherence measure. Methods: In this study, EEG signals from 17 subjects during two memory tasks were recorded. After that, these signals were preprocessed, and spectral coherence connectivity measure between pair of electrodes was computed and then compared in the five frequency bands using independent $t$-test. Results: This statistical analysis for working memory task showed significant differences in the temporal, central, and parietal lobes, especially in P7, P3, Pz, T8, C3, and C4 electrodes. As we know, these differences are related to learned skills and activities, words and sounds perception, and memory. Furthermore, for iconic memory task, significant differences were observed in the right hemisphere of these two groups. Conclusions: From this task, we can say professional pianists are different from normal people in the perception of images and creativity. Results of this study show the effects of music on human brain and memory.
\end{abstract}

Keywords: Electroencephalogram, iconic memory, spectral coherence, working memory

\section{Introduction}

Today, many researchers in medicine and engineering areas are studying biomedical signal processing, especially electroencephalogram (EEG) signal processing, which is an important part of neuroscience. Generally, EEG signal processing has been used to evaluate behavioral disorders and abnormalities in the brain. As we know, the brain has many regions, and each one of these regions has one or more important tasks, so deficiency in each region emerges as a disorder and it can be represented by EEG signals. Therefore, we can determine different brain disorders by investigating EEG signals. Furthermore, memory is an interesting aspect of neuroscience which has taken into consideration among many researchers. Memory has many types; some researchers divided it into three distinct types: short-term memory, long-term memory, and sensory memory. ${ }^{[1-3]}$ In another division, working memory is a separate system too. Working memory stores and

This is an open access journal, and articles are distributed under the terms of the Creative Commons Attribution-NonCommercialShareAlike 4.0 License, which allows others to remix, tweak, and build upon the work non-commercially, as long as appropriate credit is given and the new creations are licensed under the identical terms.

For reprints contact: reprints@medknow.com manipulates information temporarily, ${ }^{[4]}$ also it is a basic step in decision-making, acting behavior, solving problems, and reasoning. It also has important role in understanding music. ${ }^{[5]}$ However, there are many researches on working memory, but it is worthwhile. One of our goals is to investigate this worthy subject "working memory," since it is a critical part of our life, always we need to decide, for example, "what to eat?" and "what diode is appropriate for this apparatus?" In this study, we investigated EEG signals of pianists and nonpianists during working memory and iconic memory tasks. Iconic memory is part of visual sensory memory which can store high capacity of a very brief visual information.

Several studies about memory are in the field of evaluation effects of music. Schulze et $a l^{[6]}$ investigated musicians and nonmusicians during verbal and tonal working memory examinations. They recorded functional magnetic resonance imaging (fMRI) data during certain stimuli from 17 nonmusicians and 16 musicians.

\footnotetext{
How to cite this article: Boutorabi SY, Sheikhani A. Evaluation of electroencephalogram signals of the professional pianists during iconic memory and working memory tests using spectral coherence. J Med Sign Sens 2018;8:87-94.
}

\section{Seyedeh Yasamin \\ Boutorabi, Ali Sheikhani}

Department of Biomedical Engineering, Science and Research Branch, Islamic Azad

University, Tehran, Iran

Address for correspondence: Dr. Ali Sheikhani, Department of Biomedical Engineering, Science and Research Branch, Islamic Azad University, Tehran, Iran. E-mail: Sheikhani_al_81@ srbiau.ac.ir

Website: www.jmss.mui.ac.ir DOI: 10.4103/jmss.JMSS_38_17 
Then, after preprocessing steps, different types of filters were used to remove artifacts, register, normalize, and used general linear model and compute $t$-value and Z-score. Significant differences were observed between two groups in Broca's areas, presupplementary motor area (SMA)/SMA, premotor cortex, inferior parietal lobe, and left insular cortex. Furthermore, there were differences among musicians during tonal and verbal working memory examinations in regions. These differences arise from differences in training musicians and some other parameters.

Researches shown working memory store and manipulate temporary information, ${ }^{[4]}$ and is like a bridge between sensory memory and long-term memory. Furthermore, it is like a bridge between sensory memory and long-term memory. People use working memory while thinking, translating, and playing some kinds of music. They concluded that people remember rhythmic words like B, $\mathrm{V}, \mathrm{E}, \mathrm{C}$, and $\mathrm{G}$, harder than phonetic words such as $\mathrm{F}$, $\mathrm{K}, \mathrm{L}, \mathrm{R}$, and $\mathrm{X}$, since distortion and phonetic are similar. Moreover, because of this conclusion, Williamson et al., in $2010,{ }^{[7]}$ examined musicians and nonmusicians while verbal and musical stimuli. They observed that there was not any effect of phonetic similarities on remembering musicians' group. Because musician's ear trained a lot and is familiar with similar words and distortion happens less to them. ${ }^{[7,8]}$

Music can affect the structure of the brain; some researches focus on investigating effects of different types of music activities such as playing piano, this can affect the human brain. Han et al., in 2009, ${ }^{[9]}$ measured gray matter density and white matter integrity in two groups: professional pianists and normal people. This study used structural MRI and diffusion tensor MRI, and after processing brain images, these two groups observed that professional pianists had higher fractional anisotropy in their left sensorimotor cortex and right cerebellum and on the other hand had higher white matter integrity that normal people.

In this study, our aim was to measure functional connectivity from EEG signals of two groups of people, professional pianists and normal people and comparing the effects of music (playing piano) on memory. Functional connectivity computes the relationship between separate brain regions and coherence is one of the functional connectivity measures. This measure was not used in other studies of this field. Furthermore, as mentioned, studies almost used FMRI and MRI, but here we recorded our data using electroencephalography.

In this study, after recording EEG signals, we analyzed EEG standard frequency bands and then compute spectral coherence measure. Among several biomedical signal processing methods such as wavelet, empirical mode decomposition, and bispectrum, we used spectral coherence, since several studies showed that this measure generates more robust features and is suitable. ${ }^{[10,11]}$

\section{Materials and Methods}

\section{Memory tests}

As we said earlier, memory is associated with the ability of storing information for short or long terms. In this study, we used Cambridge Neuropsychological Test Automated Battery (CANTAB) examinations to measure memory. CANTAB has common examinations in neuroscience which can evaluate memory, executive function, social and emotion, attention, and psychomotor speed. It has some examinations, but in this study, we only used two examinations, the pattern recognition memory (PRM) and the spatial working memory (SWM). In the PRM examination, every time, two separate samples have been shown to the subject, and in the following step, he/she must remember the shown sample in the last step and click on a key in front of his hand [Figure 1b]. This examination is used to diagnose abnormalities and disorders at temporal lobe. ${ }^{[12]}$

In the SWM examination, a number of colored boxes are shown to the person while he must find blue boxes which till fills up the column on the right corner of the screen and continues other steps [Figure 1a]. This examination is done to evaluate the ability of people in remembering spatial information and remembering information in working memory.

\section{Database}

In this study, 17 right-handed males from 17 to 35 years old participated in the. Ten subjects for professional pianist group and seven subjects for normal group. All of these subjects were in complete mental and physical health and never used alcohol and drugs. All of them slept at a certain time at the night before the recording EEG signals.

Professional pianists were selected from people who had well skills in playing piano and they all had 5-year skill in playing piano. In the other words, all of them could simultaneously play skillfully black and white keys with their hands while pressing pedal with their feet and reading music's sheets. Also, they can remember notes and play piano very well.

Normal group was people who had no skills in any kinds of music, especially piano. These people cannot synchronously use their eyes for reading notes and their hands for playing piano.

EEG signals were recorded while participants were seated on a chair in a dark room. 19 EEG channels were recorded according to 10-20 international recording system. These signals were recorded at $128 \mathrm{~Hz}$ sampling frequency with EEG amplifier, BE Light system (EbNeuro, Italy). Reference electrodes were placed on the right and left ears. Before recordings begun, we asked from participants to be relaxed for $1 \mathrm{~min}$. EEG signals were recorded in four stages. In the first stage, EEG signals were recorded for $90 \mathrm{~s}$, whereas 
participants had closed their eyes and were seated calm on a chair. In Stage 2, participants listened carefully to a text which were related to logic memory examination (listening memory examination) and then repeated those words which listened. This stage lasts 150 s. In Stage 3, we evaluated visual memory. In this stage, participants done PRM examination while laptop was on his/her feet. This stage lasts 180 s. Moreover, in Stage 4, SWM examination done for 180 s. Figure 2 shows a participant while recording EEG signals for Stage 3 and 4. In this study, we used EEG signals of Stage 3 and 4 which belongs to PRM and SWM tests, respectively.

\section{Processing electroencephalogram time series}

Block diagram of our proposed method is shown in Figure 3. In this study, we used EEGLAB software, version 13.6.5 to process our EEG signals. After recording EEG signals, the preprocessing step begun. Despite this fact that all the participants tried to be calm during recording EEG signals, but sometimes they blinked or moved, and it was unavoidable, so we used independent component analysis (ICA) method to eliminate artifacts. ICA is a technique which can separate linearly combined sources and in this way detects and eliminates artifacts from EEG signals. This technique can be used for non-Gaussian or dependent sources. In this study, ICA detected and eliminated blink, muscles, and occipital artifacts [Figure 4]. Blink impresses prefrontal channels (Fp1 and Fp2) so ICA can detect that. If brain activities increase in occipital channels $\left(\mathrm{O}_{2}\right.$ and $\mathrm{O}_{1}$ channels $)$, ICA detects these activities and we can eliminate occipital artifacts. Moreover, muscle artifacts can be seen in temporal (T7 and T8) and close sites near that so ICA detects that. ${ }^{[13,14]}$

After this step, we used SIFT toolbox to compute spectral coherence, the functional connectivity measure. First, we detrened EEG signals using this toolbox, then fitted a model to estimate connectivity measure. There are several

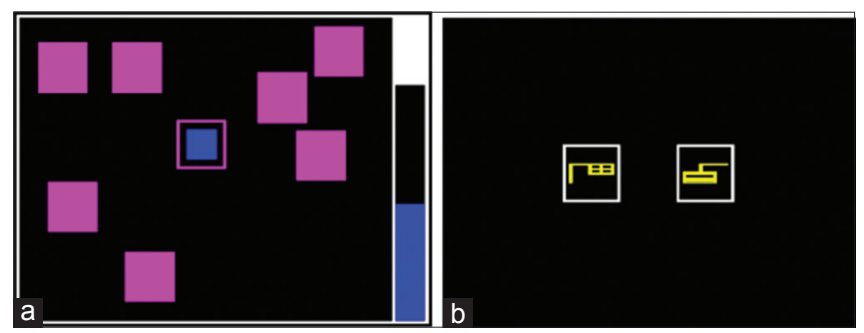

Figure 1: View of (a) A step in spatial working memory exam. (b) A step in pattern recognition memory examination

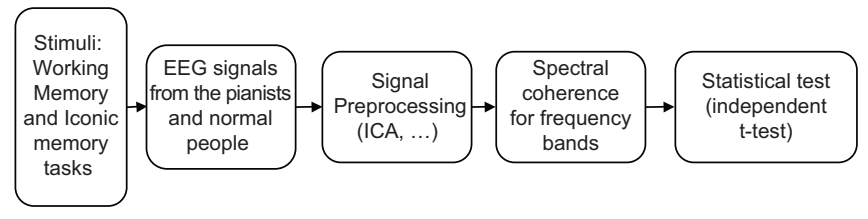

Figure 3: Block diagram of our proposed method models which can fit EEG signals; adaptive multivariate autoregressive modeling is the most common model. We chose modeling approach based on segmentation variate autoregressive. This approach is based on Fourier transformation and windowing techniques. Furthermore, we used Vierio-Morph algorithm which uses multichannel geometric mean without using least square. Here, two parameters were important: window length and window step size. We chose these parameters with try and error, window length, and window step size obtained equal to 3 and $1 \mathrm{~s}$, respectively. After that, we must choose the model order, P. We must choose model order which minimizes the information of measured criterion Schwarz-Bayes Criterion (SBC). There are two important keys in this criterion, one is the prediction of error and the other is the free parameter, which increases as model order increases. ${ }^{[15]}$ SBC (also known as Bayes Information Criterion) is computed as:

$$
\operatorname{SBC}(p)=\ln \left|\overline{\sum(p)}\right|+\frac{\ln (T)}{\hat{T}} p M^{2}
$$

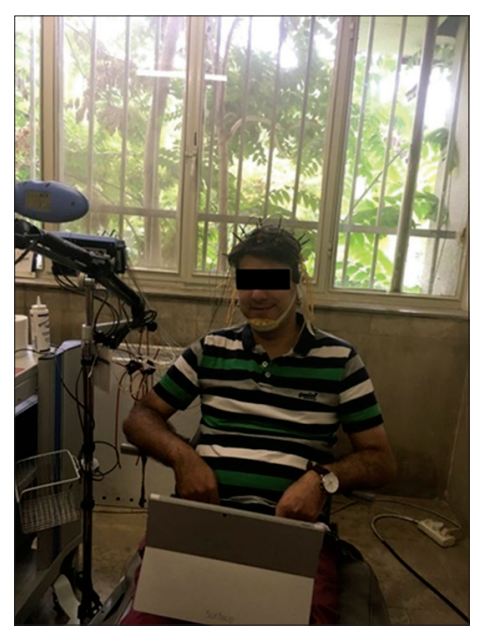

Figure 2: A participant while recording electroencephalogram signals for three and four stages

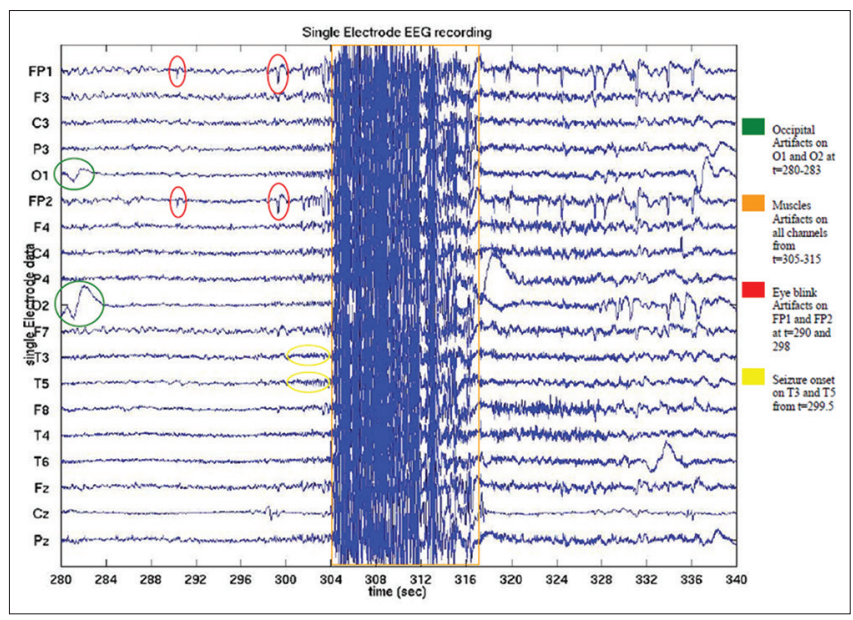

Figure 4: Independent component analysis panel and detected artifacts on electroencephalogram channels 
Where, $p$ is the model order, $\mathrm{M}$ is the number of variables, and $\hat{T}$ is the total sampling time. In this study, we tried a model order from 1 to 30 and $p$ equal to 9 or 10 and applied this criterion. Then to evaluate selected model, we used whiteness, stability, and consistency. for more information review. ${ }^{[15-17]}$ To evaluate model, we used autocorrelation function (ACF) with significant level $90 \%$ to compute whiteness. After that, we computed coherence as our connectivity measure. Then, we computed frequency bands of EEG signals: theta, delta, alpha, beta, and gamma bands to compute spectral coherence.

\section{Spectral coherence}

Coherence is some kinds of connectivity measures which calculates the similarity between two signals from different regions, in the other hand; for EEG signals, it measures the similarity between two EEG signals from different brain regions. Coherence evaluates phase similarity of two signals and if these two signals have identical phases, whether they have identical or different amplitudes, they have the most value of coherence. It can say that, coherence evaluates time or frequency stability between regions of the brain. It is said that coherence is associated with learning language, music, and planning. ${ }^{[18,19]}$

Spectral coherence is the value of matching and synchronization between two series which are in the certain frequency of $f$ For the two signals from channel $i$ and $j$, $\mathrm{COH}_{\mathrm{i}, \mathrm{j}}(f)$ is the spectral coherence between them and will be calculated from Eq. 2:[10]

$$
\mathrm{COH}_{i, j}(f)=\frac{\left|S_{i . j}(f)\right|^{2}}{S_{i . i}(f) S_{i . j}(f)}
$$

Where $S_{i, j}(f)$ or cross spectrum is the function of frequency which calculates dependency of two signals (channel $i$ and $j$ ) in time domain $S_{i, i}(f)$ and $S_{i, j}(f)$ are auto spectrum, these functions can be calculated from Welch method. Spectral coherence value is from 0 to 1, 1 denotes the highest similarity between channels and 0 denotes no similarity.

\section{Statistical test}

We used independent samples $t$-test to investigate two groups of professional pianists and normal people. This test computes mean and standard deviation of these two groups and determines that these two groups have significant differences according to a certain $P$ value or not. Before applying this test, we must test whether our data have normal distribution or not. If not, we cannot use $t$-test. To test this fact, we used KolmogorovSmirnov (K-S) test. Finally, we tested and knew the normal distribution of data, then we could apply independent $t$-test.

\section{Results}

In this section, results of two memory tasks, PRM and SWM, are shown in separate sections.

\section{Pattern recognition memory examination}

In this section, results of difference between EEG signals of the professional pianists and normal group in five frequency bands are reported as they done PRM test. Figure 5 shows connectivity results of these two groups for five frequency bands: delta, theta, alpha, beta, and gamma. As we said earlier, spectral coherence was calculated for pair of electrodes. Moreover, results of independent $t$-test are reported in Tables 1-5.

Significant pairs of electrodes in delta frequency band as results of independent $t$-test are list in Table 1. As you can see, some pair of electrodes in right hemisphere had significant differences $(P<0.05)$, and spectral coherence values of the pianist were lower than normal group.

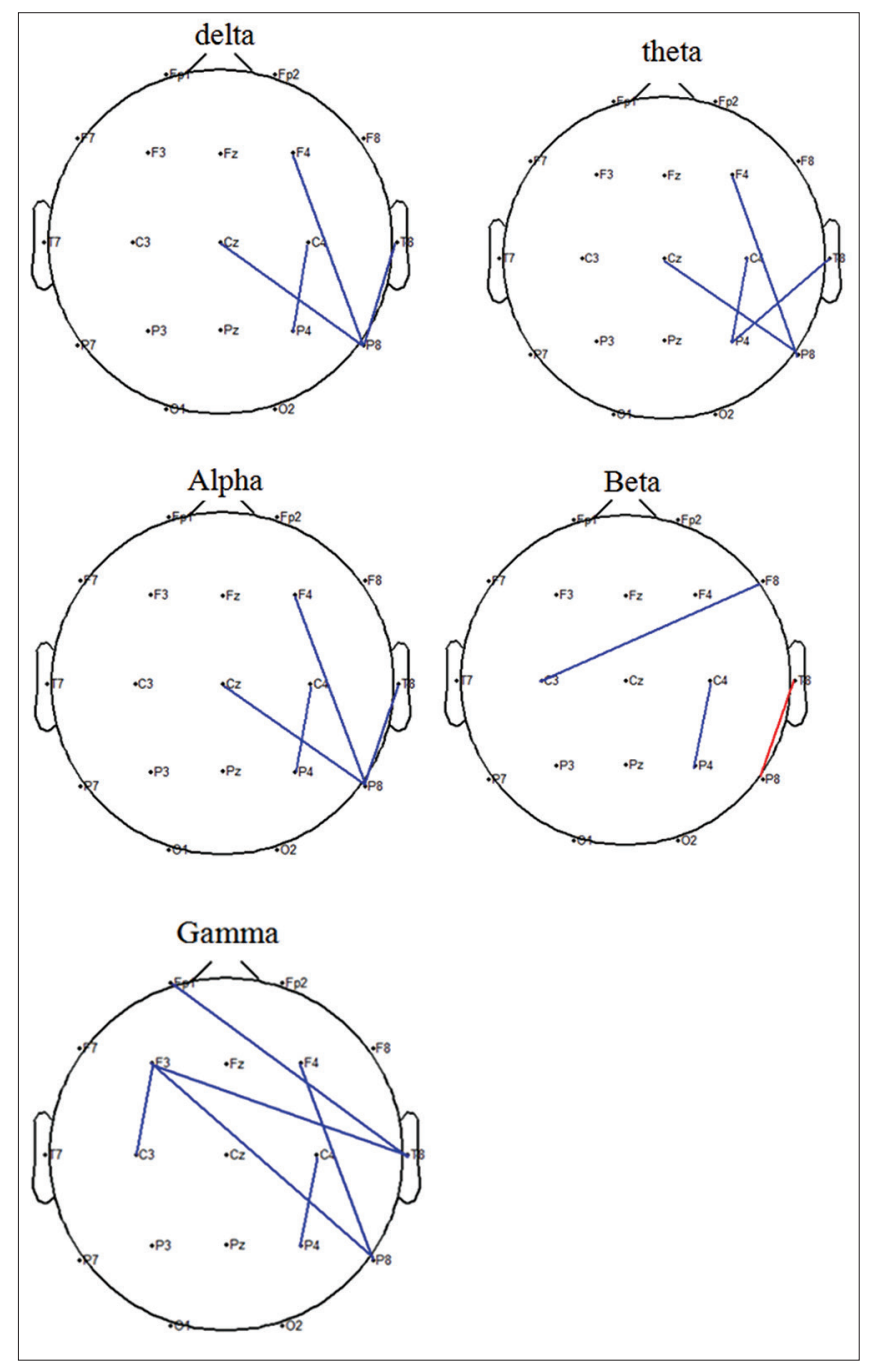

Figure 5: Spectral coherence graph between brain channels in pattern recognition memory in frequency bands. Blue color shows significant difference lower than $\mathbf{0 . 0 5}$ and red color shows significant difference lower than 0.01 
Table 1: Significant difference in pair of electrodes for delta frequency band as results of independent $t$-test

\begin{tabular}{lccc}
\hline \multirow{2}{*}{$\begin{array}{l}\text { Electrode } \\
\text { pairs }\end{array}$} & \multicolumn{2}{c}{ Mean \pm SD } & $\begin{array}{c}\text { Statistical } \\
\text { analysis }(\boldsymbol{P})\end{array}$ \\
\cline { 2 - 3 } F4-P8 & $0.487 \pm 0.048$ & $0.245 \pm 0.035$ & 0.042 \\
Cz-P8 & $0.481 \pm 0.039$ & $0.268 \pm 0.038$ & 0.049 \\
C4-P8 & $0.800 \pm 0.009$ & $0.586 \pm 0.057$ & 0.036 \\
T8-P8 & $0.616 \pm 0.042$ & $0.308 \pm 0.074$ & 0.015 \\
\hline
\end{tabular}

SD - Standard deviation

\begin{tabular}{lccc}
\hline \multicolumn{2}{l}{$\begin{array}{l}\text { Table 2: Significant difference in pair of electrodes for } \\
\text { theta frequency band as results of independent } \boldsymbol{t} \text {-test }\end{array}$} \\
\cline { 2 - 3 } $\begin{array}{l}\text { Electrode } \\
\text { pairs }\end{array}$ & \multicolumn{2}{c}{ Mean \pm SD } & $\begin{array}{c}\text { Statistical } \\
\text { analysis }(\boldsymbol{P})\end{array}$ \\
\cline { 2 - 4 } F4-P8 & $0.489 \pm 0.048$ & $0.244 \pm 0.035$ & 0.035 \\
Cz-P8 & $0.481 \pm 0.039$ & $0.268 \pm 0.037$ & 0.046 \\
C4-P4 & $0.801 \pm 0.009$ & $0.586 \pm 0.058$ & 0.028 \\
T8-P8 & $0.616 \pm 0.041$ & $0.307 \pm 0.073$ & 0.015 \\
\hline
\end{tabular}

SD - Standard deviation

\begin{tabular}{|c|c|c|c|}
\hline \multirow{2}{*}{$\begin{array}{l}\text { Electrode } \\
\text { pairs }\end{array}$} & \multicolumn{2}{|c|}{ Mean \pm SD } & \multirow{2}{*}{$\begin{array}{r}\text { Statistical } \\
\text { analysis }(P)\end{array}$} \\
\hline & Normal group & Pianist group & \\
\hline $4-P 8$ & $0.497 \pm 0.025$ & $0.242 \pm 0.034$ & 0.025 \\
\hline Cz-P8 & $0.483 \pm 0.039$ & $0.266 \pm 0.036$ & 0.040 \\
\hline C4-P4 & $0.805 \pm 0.008$ & $0.583 \pm 0.058$ & 0.027 \\
\hline Г8-P8 & $0.615 \pm 0.038$ & $0.306 \pm 0.070$ & 0.012 \\
\hline
\end{tabular}

SD - Standard deviation

\begin{tabular}{lccc}
\hline \multirow{2}{*}{$\begin{array}{c}\text { Table 4: Significant differences in pair of electrodes for } \\
\text { beta frequency band as results of independent } \boldsymbol{t} \text {-test }\end{array}$} \\
\cline { 2 - 3 } $\begin{array}{l}\text { Electrode } \\
\text { pairs }\end{array}$ & \multicolumn{2}{c}{ Mean \pm SD } & $\begin{array}{c}\text { Statistical } \\
\text { analysis }(\boldsymbol{P})\end{array}$ \\
\hline F8-C3 & $0.539 \pm 0.023$ & $0.454 \pm 0.052$ & 0.035 \\
C4-P4 & $0.813 \pm 0.016$ & $0.560 \pm 0.067$ & 0.019 \\
T8-P8* & $0.709 \pm 0.031$ & $0.335 \pm 0.049$ & 0.002 \\
\hline
\end{tabular}

$* P<0.01 . \mathrm{SD}-$ Standard deviation

\begin{tabular}{lccc}
\hline \multirow{2}{*}{$\begin{array}{l}\text { Table 5: Significant differences in pair of electrodes for } \\
\text { gamma frequency band as results of independent } \boldsymbol{t} \text {-test }\end{array}$} \\
\cline { 2 - 3 } $\begin{array}{l}\text { Electrode } \\
\text { pairs }\end{array}$ & \multicolumn{2}{c}{ Mean \pm SD } & $\begin{array}{c}\text { Statistical } \\
\text { analysis }(\boldsymbol{P})\end{array}$ \\
\hline FP1-T8 & $0.591 \pm 0.033$ & $0.363 \pm 0.067$ & 0.049 \\
F3-C3 & $0.906 \pm 0.003$ & $0.673 \pm 0.087$ & 0.036 \\
F3-T8 & $0.628 \pm 0.011$ & $0.366 \pm 0.094$ & 0.037 \\
F3-P8 & $0.484 \pm 0.047$ & $0.210 \pm 0.032$ & 0.025 \\
F4-P8 & $0.625 \pm 0.052$ & $0.339 \pm 0.049$ & 0.020 \\
C4-P4 & $0.810 \pm 0.013$ & $0.539 \pm 0.080$ & 0.017 \\
\hline SD
\end{tabular}

SD - Standard deviation

Furthermore, there was $P$ value of lower than 0.01 in P8-T8 pair electrode in beta frequency band.

Table 2 shows significant differences $(P<0.05)$ in pair of electrodes for theta frequency band. Mean spectral coherence values of pianists are lower than normal people in this frequency band too.

Table 3 shows mean and standard deviation spectral coherence values of two groups for pair of electrodes which had significant differences $(P<0.05)$ in alpha frequency band. In this band, mean spectral coherence values in professional pianist are lower than normal people.

Table 4 belongs to beta frequency band; in this table, results of independent $t$-test are shown. As you can see in Table 5, for T8-P8 pair, $P$ value was lower than 0.01 , and in the other two pairs, it was lower than 0.05. Furthermore, spectral coherence values of normal people in these pairs were higher than professional pianists.

According to Table 5, in six pairs, there were significant differences $(P<0.05)$ as results of independent $t$-test. There is a more significant pair of electrodes in gamma frequency band than other frequency bands.

In all of these tables, there were two common results: (1) significant differences in a pair of electrodes were in right hemisphere and (2) values of spectral coherence in professional pianists were lower than normal people.

\section{Spatial working memory examination}

In this section, results of spectral coherence graph and independent $t$-test in a pair of electrodes are shown while two groups were doing SWM test. Figure 6 shows spectral coherence graph for five frequency bands and Tables 6-10 list mean and standard deviation spectral coherence values and also $P<0.05$.

As shown in Figure 6, there were almost significant differences in all the parietal, central, and temporal pair of electrodes. Furthermore, significant differences were in some pair of electrodes with F3 (frontal region). There were significant differences with $P$ values lower than 0.01 in $\mathrm{P} 3-\mathrm{Pz}$ pair electrode in delta and theta frequency bands and in P3-C3 pair electrode in beta and gamma frequency bands. There were fewer significant differences in alpha frequency band in comparison to other frequency bands.

According to Tables 6-10, mean of spectral coherence values where $P$ values were lower than 0.05 for professional pianist were lower than normal people in all frequency bands, except for Fp1-T7 and Fp2-T7 pairs in gamma frequency band which were higher.

\section{Discussion}

According to results of spatial working memory examinations, there were significant differences $(P<0.05)$ in parietal, temporal, and central regions between professional pianists and normal people in all frequency bands while doing working memory test (SWM). As we know, temporal region of brain is responsible for learned skills and functions, ${ }^{[21,22]}$ and parietal region is responsible for perception of words and sounds and has 


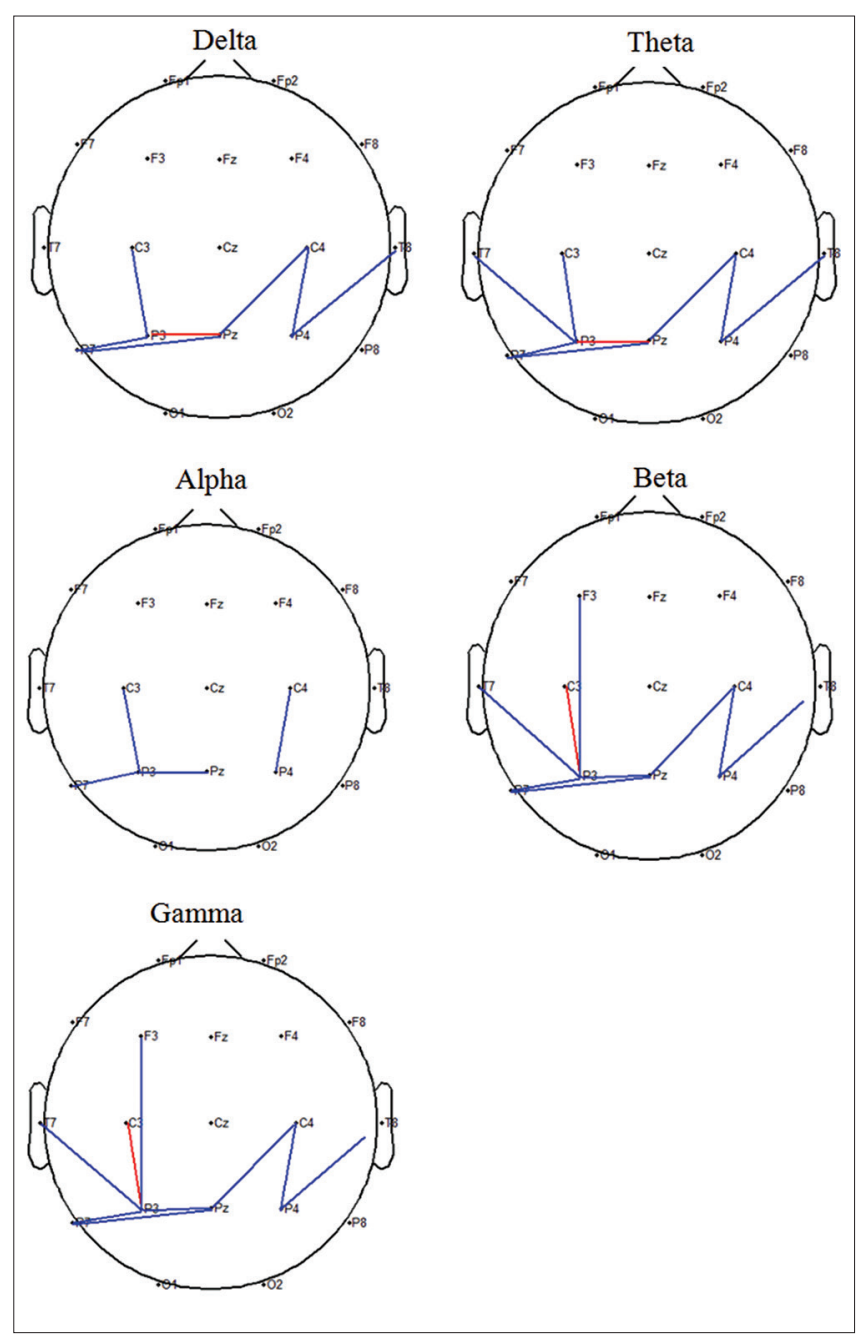

Figure 6: Spectral coherence graph between brain channels in spatial working memory in frequency bands. Blue color shows significant difference lower than $\mathbf{0 . 0 5}$ and red color shows significant difference lower than 0.01

important role in memory and intelligence. ${ }^{[23]}$ Furthermore, we have seen in Schulze study, ${ }^{[6]}$ that there are significant differences at Broca's area (in the frontal lobe of the dominant hemisphere), parietal and left hemisphere of pair of electrodes between musician and non-musician in working memory test. Moreover, in Flores-Gutiérrez et al. study, ${ }^{[24]}$ it was observed that coherence of women while listening to pleasant and annoying music had difference and related that to increased transactions in working memory, so here we can conclude that transactions in working memory are higher for a professional pianist that normal people. Furthermore, the pianist person is different from other people that have no skills in piano, as performing skills, remembering subjects, perception of sounds and words, and it is because music effects on human brain and improve its skills.

According to results of iconic memory test for of two groups, PRM, we observed significant differences among coherence values in right pair of electrodes, especially
Table 6: Significant differences in pair of electrodes for delta frequency band as results of independent $t$-test

\begin{tabular}{lccc}
\hline \multirow{2}{*}{$\begin{array}{l}\text { Electrode } \\
\text { pairs }\end{array}$} & \multicolumn{2}{c}{ Mean \pm SD } & $\begin{array}{c}\text { Statistical } \\
\text { analysis }(\boldsymbol{P})\end{array}$ \\
\cline { 2 - 3 } C3-P3 & $0.676 \pm 0.023$ & $0.403 \pm 0.058$ & 0.013 \\
C4-Pz & $0.617 \pm 0.059$ & $0.362 \pm 0.048$ & 0.050 \\
C4-P4 & $0.813 \pm 0.014$ & $0.563 \pm 0.079$ & 0.025 \\
T8-P4 & $0.658 \pm 0.028$ & $0.389 \pm 0.073$ & 0.024 \\
P7-P3 & $0.688 \pm 0.039$ & $0.400 \pm 0.048$ & 0.020 \\
P7-Pz & $0.529 \pm 0.070$ & $0.261 \pm 0.027$ & 0.040 \\
P3-Pz* & $0.730 \pm 0.015$ & $0.433 \pm 0.072$ & 0.0087 \\
\hline
\end{tabular}

$* P<0.01$. SD - Standard deviation

Table 7: Significant differences in pair of electrodes for theta frequency band as results of independent $t$-test

\begin{tabular}{lccc}
\hline $\begin{array}{l}\text { Electrode } \\
\text { pairs }\end{array}$ & \multicolumn{2}{c}{ Mean \pm SD } & $\begin{array}{c}\text { Statistical } \\
\text { analysis }(\boldsymbol{P})\end{array}$ \\
\cline { 2 - 3 } T7-P3 & $0.486 \pm 0.025$ & $0.280 \pm 0.057$ & 0.057 \\
C3-P3 & $0.677 \pm 0.023$ & $0.402 \pm 0.058$ & 0.012 \\
C4-Pz & $0.813 \pm 0.014$ & $0.562 \pm 0.079$ & 0.046 \\
C4-P4 & $0.607 \pm 0.017$ & $0.492 \pm 0.065$ & 0.025 \\
T8-P4 & $0.657 \pm 0.029$ & $0.389 \pm 0.072$ & 0.024 \\
P7-P3 & $0.688 \pm 0.039$ & $0.400 \pm 0.047$ & 0.018 \\
P7-Pz & $0.530 \pm 0.070$ & $0.261 \pm 0.026$ & 0.042 \\
P3-Pz* & $0.729 \pm 0.015$ & $0.433 \pm 0.072$ & 0.0089 \\
\hline
\end{tabular}

$* P<0.01 . \mathrm{SD}-$ Standard deviation

Table 8: Significant differences in pair of electrodes for alpha frequency band as results of independent $t$-test

\begin{tabular}{lccc}
\hline \multirow{2}{*}{$\begin{array}{l}\text { Electrode } \\
\text { pairs }\end{array}$} & \multicolumn{2}{c}{ Mean \pm SD } & $\begin{array}{c}\text { Statistical } \\
\text { analysis }(\boldsymbol{P})\end{array}$ \\
\cline { 2 - 3 } C3-P3 & $0.656 \pm 0.027$ & $0.396 \pm 0.059$ & 0.020 \\
C4-P4 & $0.784 \pm 0.015$ & $0.559 \pm 0.078$ & 0.040 \\
P7-P3 & $0.704 \pm 0.030$ & $0.400 \pm 0.046$ & 0.050 \\
P3-Pz & $0.701 \pm 0.026$ & $0.431 \pm 0.072$ & 0.022 \\
\hline
\end{tabular}

$\mathrm{SD}$ - Standard deviation

Table 9: Significant differences in pair of electrodes for beta frequency band as results of independent $t$-test

\begin{tabular}{lccc}
\hline $\begin{array}{l}\text { Electrode } \\
\text { pairs }\end{array}$ & \multicolumn{2}{c}{ Mean \pm SD } & $\begin{array}{c}\text { Statistical } \\
\text { analysis }(\boldsymbol{P})\end{array}$ \\
\cline { 2 - 3 } F3-P3 & Control group & Pianist group & 0.046 \\
T7-P3 & $0.474 \pm 0.063$ & $0.215 \pm 0.032$ & 0.023 \\
C3-P3* & $0.686 \pm 0.026$ & $0.250 \pm 0.051$ & 0.006 \\
C4-Pz & $0.642 \pm 0.052$ & $0.370 \pm 0.066$ & 0.031 \\
C4-P4 & $0.813 \pm 0.016$ & $0.577 \pm 0.038$ & 0.011 \\
T8-P4 & $0.638 \pm 0.042$ & $0.371 \pm 0.066$ & 0.032 \\
P7-P3 & $0.682 \pm 0.045$ & $0.412 \pm 0.042$ & 0.025 \\
P7-Pz & $0.551 \pm 0.069$ & $0.274 \pm 0.014$ & 0.035 \\
P3-Pz & $0.707 \pm 0.023$ & $0.412 \pm 0.076$ & 0.012 \\
\hline
\end{tabular}

$* P<0.01 . \mathrm{SD}-$ Standard deviation

P8 and T8. As we know, right hemisphere of brain is the center for perception of images and sounds and also creativity of human arises from this region. Therefore, 
Table 10: Significant differences in a pair of electrodes for gamma frequency band as results of independent

\begin{tabular}{lccc}
\hline Electrode & \multicolumn{2}{c}{$\boldsymbol{t}$-test } & \\
\cline { 2 - 3 } pairs & Control group & Pianist group & $\begin{array}{c}\text { Statistical } \\
\text { analysis }(\boldsymbol{P})\end{array}$ \\
\hline FP1-T7 & $0.398 \pm 0.075$ & $0.656 \pm 0.037$ & 0.033 \\
FP2-T7 & $0.254 \pm 0.045$ & $0.519 \pm 0.042$ & 0.045 \\
C3-P3 & $0.658 \pm 0.027$ & $0.391 \pm 0.094$ & 0.037 \\
C4-Pz & $0.637 \pm 0.051$ & $0.392 \pm 0.047$ & 0.045 \\
C4-P4 & $0.797 \pm 0.022$ & $0.516 \pm 0.067$ & 0.012 \\
\hline
\end{tabular}

$\mathrm{SD}-$ Standard deviation

we can conclude that there are differences between coherence of EEG signals of professional pianists and normal people and these arise from the difference between the ability of perception, creativity, and emotion of these two groups as results of music effects on human brain. Indeed, this study evaluated the effects of a certain skill in music, playing piano, on memory of human, maybe other music skills or the other nonmusical skills have different effects on memory, maybe it's severity or intervention be different. However, this study can help researchers into this field.

As a subject, we can say that this study evaluated average of effects of music skill (playing piano) in a number of subjects, but it needs to evaluate subject to subjects independently and consider intervariability of subjects. Future works can evaluate this problem.

\section{Conclusion}

Today, neuroscience obtained great advances and studies about evaluation effects of different factors on memory have important achievements. One of these achievements is evaluation music in human's life and its effects on behavior. In this study, we evaluate the spectral coherence of professional pianists and normal people using some memory tests: SWM and PRM. After processing EEG signals of these two groups with appropriate filters, spectral coherence values of pair of electrodes were computed and then significant differences were determined using independent samples $t$-test. Our results showed the differences between EEG signals of pianists and normal people as results of the effects of music on the brain. Furthermore, different regions between these two groups determined: temporal, parietal, and central. These regions are related to learned skills and functions, perception of words, sounds and have an important role in memory and intelligence.

\section{Declaration of patient consent}

The authors certify that they have obtained all appropriate patient consent forms. In the form the patient(s) has/have given his/her/their consent for his/her/their images and other clinical information to be reported in the journal. The patients understand that their names and initials will not be published and due efforts will be made to conceal their identity, but anonymity cannot be guaranteed.

Financial support and sponsorship

None.

Conflicts of interest

There are no conflicts of interest.

\section{References}

1. Hunt MM. The Universe Within: A New Science Explores the Human Mind. $1^{\text {st }}$ ed. Touchstone Books; 1983.

2. MacDonald M. Your Brain: The Missing Manual: The Missing Manual. Pouge Press O'Reilly Media, Inc.; 2008.

3. Cowan N. What are the differences between long-term, short-term, and working memory? Prog Brain Res 2008;169:323-38.

4. Baddeley A. Working memory: Theories, models, and controversies. Annu Rev Psychol 2012;63:1-29.

5. Schulze K, Koelsch S. Working memory for speech and music. Ann N Y Acad Sci 2012;1252:229-36.

6. Schulze K, Zysset S, Mueller K, Friederici AD, Koelsch S. Neuroarchitecture of verbal and tonal working memory in nonmusicians and musicians. Hum Brain Mapp 2011;32:771-83.

7. Williamson VJ, Baddeley AD, Hitch GJ. Musicians' and nonmusicians' short-term memory for verbal and musical sequences: Comparing phonological similarity and pitch proximity. Mem Cognit 2010;38:163-75.

8. Conrad R. Acoustic confusions in immediate memory. $\mathrm{Br} \mathrm{J}$ Psychol 1964;55:75-84.

9. Han Y, Yang H, Lv YT, Zhu CZ, He Y, Tang HH, et al. Gray matter density and white matter integrity in pianists' brain: A combined structural and diffusion tensor MRI study. Neurosci Lett 2009;459:3-6.

10. Rocca D, Campisi P, Vegso B, Cserti P, Kozmann G, Babiloni F, et al. Human brain distinctiveness based on EEG spectral coherence connectivity. IEEE Trans Biomed Eng 2014;61:2406-12.

11. Campisi P, La Rocca D. Brain waves for automatic biometric-based user recognition. IEEE Trans Inf Forensics Secur 2014;9:782-800.

12. Available from: http://www.cambridgecognition.com. [Last accessed on 2017 Jun 12].

13. Delorme A, Mullen T, Kothe C, Akalin Acar Z, Bigdely-Shamlo N, Vankov A, et al. EEGLAB, SIFT, NFT, BCILAB, and ERICA: New tools for advanced EEG processing. Comput Intell Neurosci 2011;2011:10.

14. Available from: https://www.sccn.ucsd.edu/eeglab/. [Last accessed on 2017 Jun 12].

15. Mullen TR. The Dynamic Brain: Modeling Neural Dynamics and Interactions from Human Electrophysiological Recordings (Order No. 3639187); 2014.

16. Delorme A, Mullen T, Kothe C, Akalin Acar Z, Bigdely-Shamlo N, Vankov A, et al. EEGLAB, SIFT, NFT, BCILAB, and ERICA: New tools for advanced EEG processing. Comput Intell Neurosci 2011;2011:130714.

17. Mullen TR. The Dynamic Brain: Modeling Neural Dynamics and Interactions from Human Electrophysiological Recordings. University of California, San Diego; 2014.

18. Collura T. Coherence and Brain Connectivity-Their Meaning. Available from: http://www.futurehealth.org. [Last accessed on 2016 Aug 12].

19. Srinivasan T. Entrainment and coherence in biology. Int J Yoga 2015;8:1-2. 
20. Mozaffar S, Petr DW. Artifact extraction from EEG data using independent component analysis. Information Telecommunication and Technology Center, University of Kansas, Lawrence, KS, Tech. Rep. ITTC-FY2003-TR-03050-02; December, 2002.

21. Squire LR, Zola-Morgan S. The medial temporal lobe memory system. Science 1991;253:1380-6.

22. Phelps EA. Human emotion and memory: Interactions of the amygdala and hippocampal complex. Curr Opin Neurobiol
2004; 14:198-202.

23. Kirmani MN, Garg MK, Sharma P. Parietal and occipital lobe syndromes: Neuropsychological approach. The International Journal of Indian Psychology 3:137-44.

24. Flores-Gutiérrez EO, Díaz JL, Barrios FA, Guevara MA, Del Río-Portilla Y, Corsi-Cabrera M, et al. Differential alpha coherence hemispheric patterns in men and women during pleasant and unpleasant musical emotions. Int J Psychophysiol 2009;71:43-9.

\section{BIOGRAPHIES}

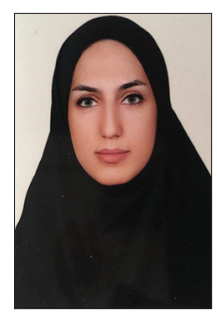

Seyedeh Yasamin Boutorabi Seyedeh Yasamin Boutorabi received a BS degree in Biomedical Engineering in 2011 from University of Isfahan, Isfahan, Iran and her MS degree in Biomedical Engineering from Islamic Azad University Science and Research Branch, Tehran, Iran in 2017. Her current research interests are in the field of Biomedical Signal Processing, Computational neuroscience and EEG Signal Processing.

Email: yasi.boutorabi@gmail.com

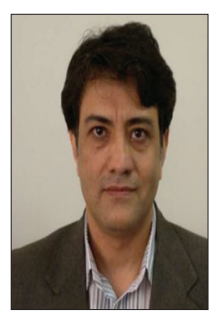

Ali Sheikhani Ali Sheikhani received his $\mathrm{PhD}$ degree in Biomedical Engineering in 2009 from Islamic Azad University Science and Research Branch, Tehran, Iran. He is now a $\mathrm{PhD}$ scholar in biomedical engineering department in Islamic Azad University, Science and Research Branch, Tehran. He is expert in biomedical signal processing and modelling, advanced signal processing and data analysis, bio instruments.

Email: sheikhani_al_81@srbiau.ac.ir 